\title{
Benign Acute Childhood Myositis
}

\author{
Fatma Müjgan Sonmez ${ }^{a}$ Murat Çakir ${ }^{b}$ Şeker Yaylab Cavit Boz ${ }^{\mathrm{c}}$ \\ Departments of a Child Neurology, ${ }^{\mathrm{b} P a e d i a t r i c s}$ and ${ }^{\mathrm{c}}$ Neurology, Faculty of Medicine, \\ Karadeniz Technical University, Trabzon, Turkey
}

\section{Key Words}

Myositis · Childhood

\begin{abstract}
Objective: To evaluate the clinical and laboratory findings of benign acute childhood myositis. Subjects and Methods: Six children, 1 girl and 5 boys, aged 3-9 years, were admitted to Karadeniz Technical University Paediatric Neurology Department in Trabzon, Turkey, with the diagnosis of benign acute childhood myositis in January and February 2002. The clinical and laboratory findings on the patients were analysed. Results: Serum creatine kinase and aspartate aminotransferase levels were increased in all cases. Two patients had leucopenia. Viral studies were negative. All the patients had a good outcome, and full recovery was achieved within $12 \mathrm{~h}$ to 3 days. Conclusion: Benign acute childhood myositis is a self-limiting disease with a good prognosis. Pediatricians and pediatric neurologists must be aware of this condition to avoid unnecessary investigations and to differentiate this condition from other causes of acute onset of inability to walk. It may occur in epidemics mainly in the wintertime, suggesting a viral aetiology.
\end{abstract}

Copyright ( 92004 S. Karger AG, Basel

\section{Introduction}

Benign acute childhood myositis (BACM) is a well-recognized disease, first described in 1957 by Lundberg [1] who studied 74 children afflicted with the disease. BACM is a benign, self-limiting condition affecting school-age children (usually boys) and is characterized by calf tenderness and the sudden onset of difficulty in walking after a viral illness, sometimes associated with influenza epidemics in the wintertime. Serum creatine kinase is elevated in the vast majority of affected patients $[2,3]$.

We report 6 cases of BACM occurring in a short period in our area and discuss the epidemiology, clinical findings, diagnosis and treatment of the condition.

\section{Subjects and Methods}

Six children, 1 girl and 5 boys, aged between 3 and 9 years (mean age 5.9 years), were admitted to Karadeniz Technical University Paediatric Neurology Department during January-February 2002 with a clinical picture of BACM. None of the children had been subjected to trauma or injections, nor did they exhibit any signs suggestive of systemic immune complex disease, such as arthritis, skin rash, prolonged fever or obvious nephritis. None presented a family history of muscle disease or reported a change in the colour of urine.

Full physical and neurological examination was performed on all patients. Levels of haemogram and serum creatine kinase (CK), aspartate aminotransferase (AST), alanine aminotransferase (ALT) were measured in all patients at admission, on the 2 nd and 4th days, and at the end of 1 week. Hepatitis markers, antinuclear antibody

\begin{tabular}{ll}
\hline KARGER & ( ) 2004 S. Karger AG, Basel \\
Fax +4161306 1234 & 1011-7571/04/0134-0227\$21.00/0 \\
$\begin{array}{l}\text { E-Mail karger@karger.ch } \\
\text { www.karger.com }\end{array}$ & $\begin{array}{l}\text { Accessible online at: } \\
\text { www.karger.com/mpp }\end{array}$
\end{tabular}

F. Müjgan Sonmez

Department of Child Neurology, Faculty of Medicine

Karadeniz Technical University

TR-61080 Trabzon (Turkey)

Tel. +90 462 3775617, Fax +90 462 3252270, E-Mail fmaynacif@dr.com 
Table 1. Clinical features of six patients with acute myositis

\begin{tabular}{llrllllll}
\hline $\begin{array}{l}\text { Pa- } \\
\text { tients }\end{array}$ & Age/sex & $\begin{array}{l}\text { Prodromal } \\
\text { period, day }\end{array}$ & $\begin{array}{l}\text { Initial CK } \\
\text { U/l }\end{array}$ & $\begin{array}{l}\text { CK after } \\
1 \text { week, U/l }\end{array}$ & $\begin{array}{l}\text { AST/ALT } \\
(5-40 \mathrm{U} / \mathrm{l})\end{array}$ & $\begin{array}{l}\text { WBC } \\
\mathrm{mm}^{3}\end{array}$ & EMG & $\begin{array}{l}\text { Viral studies } \\
\text { (NPA) }\end{array}$ \\
\hline 1 & $9 / \mathrm{M}$ & 3 & 6,424 & 110 & $480 / 312$ & 4,900 & normal & negative \\
2 & $3 / \mathrm{F}$ & 20 & 206 & 107 & $52 / 16$ & 6,400 & not done & $\begin{array}{l}\text { not done } \\
3\end{array}$ \\
$3.5 / \mathrm{M}$ & 4 & 712 & 80 & $64 / 24$ & 6,400 & not done & not done \\
4 & $8 / \mathrm{M}$ & 5 & 7,681 & 147 & $306 / 62$ & 3,100 & not done & not done \\
5 & $6 / \mathrm{M}$ & 3 & 18 & 105 & $210 / 16$ & 4,600 & normal & not done \\
6 & $6 / \mathrm{M}$ & 3 & 3,458 & 125 & $69 / 23$ & 3,100 & not done & negative \\
\hline
\end{tabular}

(ANA), anti-DNA, anti-streptolysin-O (ASO), C-reactive protein (CRP), latex, Toxoplasma, Cytomegalovirus and rubella IgM and IgG, and cold agglutinins for Mycoplasma pneumonia were also studied in all patients. Nasopharyngeal aspirate (NPA) for influenza A and B, parainfluenza type 1, 2 and 3, adenovirus, respiratory syncytial virus were studied in only 2 children. Electromyography was performed on only 2 patients and lumbar puncture in 1 who had decreased tendon reflexes.

\section{Results}

The clinical manifestations in all cases were similar. There was a prodromal illness 3-20 days (mean 6.3 days) before the onset of pain and weakness, which consisted of fever and flu-like illness with upper respiratory tract symptoms. Acute onset of symmetrical muscle pain with some weakness and tenderness involving both calves (gastrocnemius) with inability to walk were the presenting symptoms in all cases on admission. Muscle strength was normal in all cases. One patient had decreased deep tendon reflexes. Other neurological signs were normal in all of the patients.

At the time of presentation, all the patients were afebrile, 2 patients had transient leucopenia while the rest showed a marginally low peripheral white blood cell count; all had predominant lymphocytosis. Haemoglobin and platelet count and urine analysis were negative in all patients. Serum CK levels were increased with values ranging from 206 to 7,681 U/1 (normal; 30-170 U/1) at admission (table 1). All patients had elevated serum AST levels, however only 2 patients had elevated serum ALT levels. Other routine investigations, which included urea, electrolytes, calcium, phosphate, glucose and protein, were normal. Hepatitis markers, ANA, anti-DNA, ASO, CRP, Latex, Toxoplasma, Cytomegalovirus and rubella IgM and IgG, and cold agglutinins for Mycoplasma pneumonia were negative. Urine, blood, throat cultures were also obtained from 3 children and were all negative. Naso- pharyngeal aspirate for influenza A and B, parainfluenza types 1, 2 and 3 , adenovirus and respiratory syncytial virus were negative for 2 patients. Cerebrospinal fluid was obtained only in 1 patient who had decreased deep tendon reflexes and was normal. Electromyography was performed in 2 patients and no abnormality was described. Clinical recovery in all patients occurred between $12 \mathrm{~h}$ and 3 days. Subsequent estimation of CK, AST and ALT levels showed normal results after 1 week.

\section{Discussion}

BACM is a transient, self-limited disorder of mid childhood, typically affecting boys $[2,3]$. The pathogenesis is still unclear but may be an inflammatory process of muscles following direct viral invasion or induced by an immune-mediated mechanism [2]. There are some clues for a viral aetiology, such as typical prodromal illness, high prevalence in winter and spring, haematological findings and positive viral studies in some patients. Many viruses have been implicated, such as influenza, especially type B, adenovirus, parainfluenza [4, 5]. Belardi et al. [6] reported a case associated with $M$. pneumonia infection.

The mean age for BACM is 9.2 years [4]. Only 4 adult cases with BACM have been reported in the literature [1]. In our series, the mean age was 5.9 years; the age range was between 3 and 9 years with male dominance. This age range and dominance of males are similar to a previous report [1]. The frequent incidence in males is not explained, but onset of calf pain after strenuous exercise in the previous day showed that the increased incidence in males may be related to greater levels of activity compared with females [2].

In the prodromal period, there was a pain-free interval 1-7 days after a respiratory illness, followed by calf myalgia. Other symptoms were headache, nausea, vomiting, 
anorexia, abdominal pain and skin rash, as reported in the literature [7]. In our study, the period between prodromal illness and myositis was 3-20 days, compared to 2-31 days in previous studies [2]. All of our patients had flulike prodromal symptoms.

The clinical picture of patients in this study is almost typical: acute onset of symmetrical calf muscle pain and tenderness, weakness of mainly the lower extremities, and inability or refusal to walk $[2,3]$. Sometimes other muscle groups were involved such as the thighs, back, neck and proximal arm muscles [2]. Normal muscle strength is present in the majority of patients. Sensory deficits are lacking, and reflex changes are rarely noted [6]. The clinical presentation and physical findings of our patients were similar to those reported in the literature.

The most striking and pathogonomic laboratory finding of BACM are the elevated CK levels [2, 3], which typically return to normal within 1 or 2 weeks [3]. A transient elevation of AST and ALT has also been reported. In our series, all of the cases had elevated CK and AST levels, and 2 had elevated ALT levels [2]. Leukopenia is commonly observed in BACM. Our 2 patients had leukopenia, while the rest had a mild decrease in white blood cells $[2,6]$. The laboratory recovery is usually within 1 week [2, 3]. Recurrent episodes were described in a few patients. In our series, laboratory recovery was obtained after clinical recovery within 1 week and no recurrent episodes were seen.

BACM episodes may be epidemic and the majority of these episodes (approximately 60\%) occur in the winter $[2,3]$. In most reported series, myositis occurred concurrently with an influenza epidemic in the community and was caused by the same viral strain $[5,8,9]$. There are no epidemiological data about influenza or other viruses in our country. We could only study NPA for influenza A and $B$, parainfluenza type 1,2 and 3 , adenovirus and RSV in 2 of our patients, all of which were found to be negative.
In spite of this, we think that the seasonal pattern and the clinical progression of our cases support a viral aetiology, and the fact that 6 cases occurred in a short period verifies the epidemic nature of our cases.

It is important to differentiate BACM from more serious causes of refusal to walk, such as diffuse myalgia of influenza, viral myositis associated with myoglobinuria, childhood idiopathic polymyositis, dermatomyositis, acute diffuse rhabdomyolysis, trichinosis, acute cerebellar ataxia and Guillain-Barré syndrome [1-7]. Most of these can be excluded by a careful physical examination and laboratory findings $[4,6]$, with Guillain-Barré syndrome being the most important to eliminate. Normal muscle power and reflexes in most cases combined with elevated $\mathrm{CK}$ are the major findings in distinguishing BACM from Guillain-Barré syndrome. It is also important to differentiate BACM from viral myositis associated with myoglobinuria (toxic myoglobinuria type II) because it carries a high mortality and needs more urgent and aggressive supportive therapy. In toxic myoglobinuria, patients appear very ill and febrile, and present with leucocytosis and dark urine. In our cases, urine colour was normal and the patients were in a good general condition.

\section{Conclusion}

This study shows that BACM presents with a typical clinical picture and laboratory findings such as sudden onset of gait abnormality a few days after flu-like illness and raised CK. It tends to occur in epidemics during the months of January and February, and has a good outcome in uncomplicated cases. Pediatricians and pediatric neurologists must be aware of this condition to differentiate it from other cases of acute onset of inability to walk and to avoid unnecessary investigations, as well as to enlighten parents about this disease in the wintertime.

\section{References}

Childhood Myositis
1 Lundberg A: Myalgia cruris epidemica. Acta Paediatr Scand 1957;46:18-31.

2 Mackay MT, Kornberg AJ, Shield LK, Dennett $\mathrm{X}$ : Benign acute childhood myositis. Laboratory and clinical features. Neurology 1999;53: 2127-2131.

3 Zafeiriou DI, Katzos G, Gombakis N, Kontopoulos EE, Tsantali C: Clinical features, laboratory findings and differential diagnosis of benign acute childhood myositis. Acta Paediatr 2000;89:1493-1494.

4 Hassoon MM, Qandalji BR, Eeg-Olofsson O, Heijazi Z: Benign acute childhood myositis in Kuwait. Ann Trop Paediatr 1992;12:311-315.
5 Karpathios T, Kostaki M, Drakonaki S, Garoufi A, Siahanidou S, Spirou N, Theodoridis C: An epidemic with influenza B virus causing benign acute myositis in ten boys and two girls. Eur J Pediatr 1995;154:334-336.

6 Belardi C, Roberge R, Kelly M: Myalgia cruris epidemica (BACM) associated with Mycoplasma pneumonia infection. Ann Emerg Med 1987;16:579-581.

7 Antony JH, Procopis PG, Ouvrier RA: Benign acute childhood myositis. Neurolgy 1979;29: 1068-1071. 